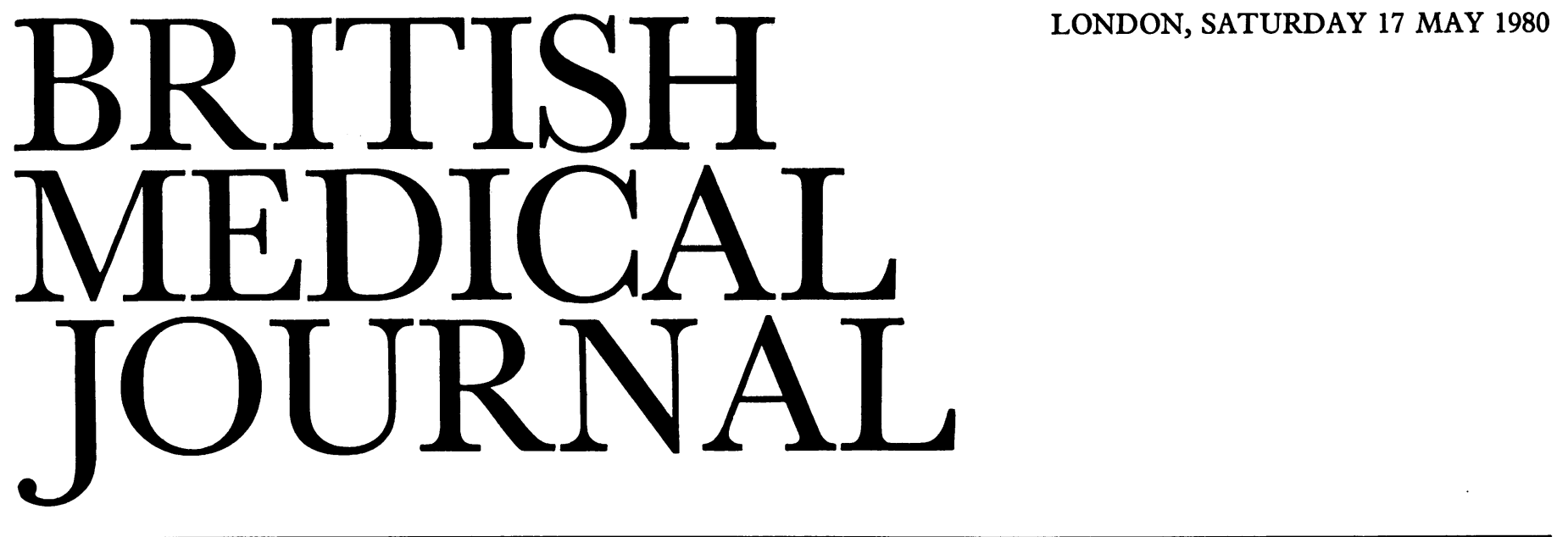

Current regulations on the use of antibiotics in animals bred for food have failed to prevent the rapid emergence of multiple drug resistance in Salmonella typhimurium in bovines in Britain. Presenting convincing evidence for this, Threlfall and his colleagues ( $p$ 1210) show that two types of multiresistant salmonellas that first appeared in bovines in 1977 were identified in 290 cases of human salmonellosis in Britain up to the end of 1979. During 1979 a new variant appeared and affected calves on at least 50 farms in the south and south-west of England, spreading to Cambridgeshire and Yorkshire through the distribution of infected animals. This latest multiply-resistant strain was induced by the new use of trimethoprim in bovines. Twenty human infections with this strain had been identified by the end of 1979. These developments must be a cause of real concern to doctors: the latest variant is resistant to ampicillin, chloramphenicol, kanamycin, streptomycin, sulphonamides, tetracyclines, trimethoprim, and furazolidone. If such a strain causes septicaemia or other extraintestinal lesions in a patient most antibiotics will be ineffective. In human salmonellosis caused by the first two of the multiresistant types most of the patients suffered only mild-to-moderate enteritis, but some had severe diarrhoea persisting for several weeks; one elderly patient died, seven had invasion of the blood stream, and one 3-year-old infant died of septicaemia. Obviously we cannot dismiss the problems as unimportant, particularly as cases have not been confined to farming families and the multiresistant strains have evidently entered the food chain.

Legislation was enacted, as a result of the Swann Report, ${ }^{1}$ precisely to prevent such developments. Antimicrobial substances (mainly antibiotics) were classified into two categories, "feed" and "therapeutic." Those in the feed category had either no therapeutic role or very little, and were available for use in animal feeds without prescription. Therapeutic antimicrobials could be prescribed only by a medical or a veterinary practitioner, and the regulations emphasised that the veterinarian was to prescribe a therapeutic antimicrobial only if he had the animals under his care. Suggestions that chloramphenicol might be prohibited from veterinary use were resisted; they were not recommended by the Swann Report on the grounds that strict limitations on its use were to be set out and that these would be observed by a responsible profession anxious to maintain good ethical standards. In an earlier paper ${ }^{2}$ Threlfall and his colleagues suggested that the veterinary profession ought to show more prudence in its prescribing habits; and in their present paper they say that "the responsibility to prevent or control drug resistance in $S$ typhimurium in bovines in Britain lies with the veterinary profession." But this oversimplifies the matter: members of the medical profession also must accept reproach for their uncritical prescribing of antibiotics. Richmond and Linton $^{3}$ conclude that the medical as opposed to the veterinary use of tetracycline seems to have created the main selective pressure for the high incidence of tetracycline-resistant organisms in the human population.

Apart from incautious prescribing, we must look at other factors in seeking to discover why the Swann recommendations have failed in their main objective-namely, preventing the spread of resistant micro-organisms from farm animals to man. The Swann Committee laid considerable emphasis on preserving the therapeutic usefulness of chloramphenicol, and the great increase in chloramphenicol resistance in the agricultural environment is particularly worrying. There are several reasons for the failure. Firstly, the use of other drugs-such as tetracycline, trimethoprim, and probably others used in animals-may encourage the spread of resistance to chloramphenicol as part of the multiple resistance carried by some of the organisms the antibiotics are directed against. Secondly, over-enthusiastic representatives of pharmaceutical firms as well as black market operators may find farmers, including poultry producers, all too ready to sidetrack their veterinarians and to bid for any supplies of prescription-only antibiotics that may become available through irregular channels. Prosecutions may close that door if evidence is forthcoming, and farmers need to be educated out of attempting to diagnose and treat or prevent enteritis by using antibiotics without veterinary help. Thirdly, advertisements in farming papers encourage such attitudes among farmers; this trend needs to be reversed, however delicately and indirectly the hint is conveyed. Fourthly, the long-incubated protein-processing order should have been introduced well before now and made law to reduce salmonella-infected feeds given to animals.

We need an administrative and political reaction to the regrettable discovery that-for whatever reason-the limited regulations based on the Swann Report have failed. Exactly why they failed, and when, may not be easy to discover; but 
clearly the present state of affairs is unsatisfactory and dangerous and more stringent regulations are needed.

1 foint Committee on the Use of Antibiotics in Animal Husbandry and Veterinary Medicine (Swann Report). Cmnd 4190. London: HMSO, 1969.

2 Threlfall BJ, Ward LR, Rowe B. Spread of multiresistant strains of Salmonella typhimurium phage types 204 and 193 in Britain. $\mathrm{Br} \mathrm{Med} \mathcal{F}$ 1978;ii:997.

${ }^{3}$ Richmond $\mathrm{MH}$, Linton KB. The use of tetracycline in the community and its possible relation to the excretion of tetracycline-resistant bacteria. $\mathcal{f}$ Antimicrob Chemother 1980;6:33-41.

\section{Reactive arthritis}

Reactive arthritis is defined as an aseptic arthritis closely related to an episode of infection elsewhere in the body. Bacterial enteritis is one well-known cause, non-gonococcal urethritis with putative organisms is another; but in many cases no source of infection is identified. Reiter's syndrome is a particularly florid form with mucocutaneous and ocular lesions, but the use of this term has led to the inelegant "incomplete Reiter's syndrome" for the more common but less florid cases. The synovitis usually starts acutely and is frequently asymmetrical, with the knees and ankles most commonly affected. Often there are also inflammatory lesions of tendon sheaths and entheses, such as plantar fasciitis. A careful examination will often elicit unsuspected pain and symmetrical limitation of movement of the lumbar spine. Radiographs at this stage are often normal. The severity and duration of the acute episode are extremely variable, as is the likelihood of recurrence, but a prolonged and stormy course is more likely in individuals with certain histocompatibility antigens, particularly HLA-B27. ${ }^{1}$

Reactive arthritis often occurs in association with enteritis caused by salmonellas and certain shigella strains ${ }^{2}$ and in Scandinavia particularly and occasionally in Britain with yersinia infections; some forms of campylobacter enteritis may also induce the reaction. ${ }^{34}$ These forms of reactive arthritis may occur in prepubertal patients without sexual contact, and in males urethritis is a frequent component of these non-sexually acquired forms. A strong association of promiscuous sexual contact surrounds reactive arthritis and Reiter's syndrome, perhaps because urethritis is a common feature and cases occur most frequently in the sexually active years, but the evidence is circumstantial. Even in patients with recent sexual contacts other known causes of infection should be excluded before making a diagnosis of sexually acquired reactive arthritis. Sex and reactive arthritis may have become too closely associated in the minds of physicians. For example, ${ }^{5}$ a 28-year-old man developed dysuria, urethritis, and reactive arthritis after an episode of fever and diarrhoea, with appreciably raised serum concentrations of antibody to Yersinia enterocolitica; "he absolutely denied any recent sexual contacts." Little information on promiscuity is available in age-matched and sex-matched controls, and a recent study of sexually acquired reactive arthritis failed to elicit a history of recent contacts with new partners in $17 \%$ of the patients, and not all urethral reinfections led to recurrences of reactive arthritis. ${ }^{6}$ Chlamydias and ureaplasmas may be isolated from the urethra or cervix of patients with reactive arthritis and antibodies to these organisms may be found, but the frequency of isolation is not greatly different from that in patients with urethritis without reactive arthritis, in whom antibodies may also be present-though often in lower titres. In short, Koch's postulates remain unfulfilled for a "transmissible agent." Possession of the antigen HIA-B27 affects severity and prognosis, but reactive arthritis is not confined to persons with it. Keat $e l$ al found that rather more of their patients with sexually acquired reactive arthritis presenting in the rheumatology clinic carried B27 than those in the venereology clinic.

The clear links between reactive arthritis and bacterial infection may provide useful clues to the mystery of rheumatoid arthritis and other connective tissue diseases. In some forms of reactive arthritis the mechanisms have been partially unravelled. Several forms of meningococcal arthritis occur with meningococcal meningitis, one being an aseptic or reactive arthritis in which meningococcal antigen and antibody are found in the blood and may subsequently be shown within polymorphs (together with complement) in affected joints. ${ }^{7}$ That an aseptic or reactive arthritis may accompany proved gonococcal urethritis has long been known, and this form of non-bacterial "gonococcal" arthritis is more common in Britain than in the United States, where classical gonococcal arthritis is found as a complication of more severe and chronic gonococcal pelvic infection. A recent report by $L$ Rosenthal and B Olhagen at the Fourth Congress of the South-east Asia and Pacific Area League against Rheumatism in 1980 strengthened the view that some cases of postgonococcal arthritis may be a true reactive arthritis rather than one in which the sterile cultures of the joint fluid were merely false-negative results. Careful prospective studies on the lines used by Greenwood ${ }^{7}$ in his studies of meningococcal arthritis may elucidate the mechanisms of induction in reactive and other forms of arthritis. Identification in affected joints of some fragment or amino-acid sequence specific to chlamydias or ureaplasmas might allow us to conclude that sexually acquired reactive arthritis is in fact sexually acquired.

1 Bitter $T$, ed. Reiter's syndrome. A symposium edited by $T$ Bitter. Ann Rheum Dis 1979 ;38 :suppl.

2 Masi AT. Epidemiology of B27-associated diseases. Ann Rheum Dis $1979 ; 38$ :suppl I 131-4.

3 Berden JHM, Muytjens HL, van de Putte LBA. Reactive arthritis associated with Campylobacter jejuni enteritis. $\mathrm{Br}$ Med $\mathcal{F}$ 1979;i:380-1.

4 Urman JD, Zurier RB, Rothfield NF. Reiter's syndrome associated with Campylobacter fetus infection. Ann Intern Med 1977;86:444-5.

5 Ford DK. Yersinia-induced arthritis and Reiter's syndrome. Ann Rheum Dis 1979;38:suppl I 127-8.

- Keat AC, Maini RN, Pegrum GD, Scott JT. The clinical features and HLA associations of reactive arthritis associated with non-gonococcal urethritis. $Q \mathcal{F}$ Med 1979;48:323-42.

7 Greenwood BM, Whittle HC. In: Dumonde DC, ed. Infection and immunology in the rheumatic diseases 1976. Oxford: Blackwell, 1976: 119-27.

\section{Patellar tendon rupture}

Traumatic rupture of the patellar tendon occurs in the young and is caused by sudden contraction of the quadriceps against resistance. ${ }^{1}$ The lesion usually occurs at the point where the patellar tendon (sometimes less properly called the patellar ligament) is attached to the lower pole of the patella, and less commonly at its tibial attachment. Apart from injuries caused by cutting with sharp objects such as glass or metal (and in one instance a needle during diagnostic amniocentesis) ${ }^{2}$ a tear through the substance of a normal tendon does not occur. ${ }^{3}$ 